\title{
Is CSMA Able to Coordinate Multiple Access in Vehicular Radio Channels Effectively?
}

\author{
Jens Mittag and Hannes Hartenstein \\ Institute of Telematics \& Steinbuch Centre for Computing \\ Karlsruhe Institute of Technology, Germany \\ Email: $\{$ jens.mittag, hannes.hartenstein $\} @$ kit.edu
}

\begin{abstract}
The first generation of inter-vehicle communication networks will most likely be based on the IEEE 802.11p standard. That is, they are going to deploy Carrier Sense Multiple Access (CSMA) to coordinate channel access among neighboring vehicles. Recently, concerns have been raised that fast-fading propagation conditions, i.e. a time- and frequency-selective fading as reported by several measurement campaigns in highway environments, might challenge the effectiveness of CSMA. These concerns also lead to the situation that alternative medium access control solutions are being discussed in standardization bodies. In this paper, we evaluate whether these concerns are justified or not. In comparison to previous studies, we use a high fidelity network simulator to study the extent by which the effectiveness of CSMA is reduced if such fading propagation conditions are considered. We also resolve the two reasons that may cause incoordination - either simultaneous transmission times or hidden terminal situations - and conclude that CSMA is able to effectively coordinate multiple access in vehicular radio channels as long as the load offered to the channel does not approach the maximum capacity.
\end{abstract}

\section{INTRODUCTION}

According to current research efforts and standardization activities, the first generation of safety-related inter-vehicle communication networks is going to employ the well known and widely studied IEEE $802.11 \mathrm{p}$ standard specification for wireless access in vehicular environments. IEEE 802.11p uses an Orthogonal Frequency Division Multiplexing (OFDM) based physical layer and a Carrier Sense Multiple Access (CSMA) based medium access control (MAC) scheme to provide robust and reliable communications in the $5.9 \mathrm{GHz}$ frequency band. However, CSMA and the physical layer of IEEE $802.11 \mathrm{p}$ have not been designed for a usage in vehicular radio channels in the first place, and concerns have been raised that CSMA might not be suited to coordinate multiple access effectively in all situations. The apparent question is therefore: are these concerns justified, and is the IEEE 802.11p MAC able to support reliable and robust safety-related inter-vehicle communications?

Over the past years, results of several channel measurement campaigns have been reported in the wireless channel modeling community, cf. [1] for an extensive survey. According to the authors of these papers, the wireless vehicular channel at $5.9 \mathrm{GHz}$ is time- and frequency-selective - in contrast to the stationary wireless indoor channel - which means that the impulse response of the channel is varying significantly with respect to frequency and over time. Subsequent physical layer evaluations have further shown that the bit error and packet delivery ratios suffer significantly if these fading characteristics are present. Will CSMA be affected in the same negative way? Is the "listen before talk" principle going to be challenged, in the sense that neighboring nodes will not be able to successfully sense ongoing transmissions within the close surrounding? If the answer to this questions is "yes", CSMA might not be able to suppress concurrent transmissions by neighboring nodes effectively.

In this paper, we evaluate whether the raised concerns are justified. We use a basic highway scenario, in which timeand frequency-selective channel conditions are pronounced, and characterize how well CSMA is able to coordinate the periodic broadcasting of beacon (or status) messages between all nodes. In particular, we assess the influence of fading radio propagation conditions on CSMA's ability to suppress concurrent packet transmissions by neighboring nodes. Compared to previous work, e.g. [2], [3], [4], which employed network simulators that abstract the microscopic details of the physical layer and the wireless channel, we base our work on a high fidelity network simulator [5]. The employed network simulator emulates the signal processing steps at the physical layer and the multi-path radio propagation effects observable on the wireless channel. The results of our simulation-based assessment indicate that

1) fading channel effects have only a slight impact on the effectiveness of CSMA,

2) interfering transmissions originate primarily from outside the so called (deterministic) carrier sense range, and

3) channel congestion is the primary issue for inter-vehicle communications.

The rest of this paper is structured as follows: Section II provides a brief overview on the related work, followed by an introduction to inter-vehicle communication channels and their influence on the physical layer performance of IEEE $802.11 \mathrm{p}$ in Section III. The evaluation of CSMA's coordination performance - including a description of CSMA, the applied evaluation method and the obtained results - is then presented in Section IV. Section V eventually concludes this work.

\section{RELATED WORK}

The performance of CSMA-based medium access control in broadcast radio channels has extensively been studied in 
the past, either analytically or empirically by means of simulations. Due to space restrictions and their lack of accuracy in terms of fading radio channel effects, or due to their focus on unicast data traffic instead of broadcast traffic, the analytical contributions, e.g. Tobagi et al. [6], Bianchi [7], or Gupta and Kumar [8], are skipped in the following.

The coordination performance and broadcast reception rates of IEEE $802.11 \mathrm{p}$ were studied by Torrent-Moreno et al. in [2], [9] using a simulation-based approach and the example of a highway scenario. The authors developed a metric denoted as Packet Level Incoordination and quantified the probability that a node interfered with an ongoing transmission of a neighboring node due to the hidden terminal problem. The proposed metric is adopted in this work, slightly generalized to include incoordinations that occur due to identical transmission times, and re-evaluated using a more accurate wireless channel and physical layer representation.

Bilstrup et al. compared the effectiveness of IEEE 802.11p and Self-Organizing TDMA (STDMA) in [3] and evaluated the distance between a transmitting and an interfering node whenever incoordinated transmissions were observed in the conducted simulations. According to the shown results, incoordinated transmission are rarely observed from close distances when STDMA is used, whereas incoordinated transmissions from close distances have to be excepted in case of CSMA. However, the authors did not evaluate how often such incoordinated transmission happened, and further did not consider fading channel conditions.

Recently, Schmidt-Eisenlohr et al. studied the impact of interference in CSMA-based inter-vehicle communication networks [10]. Their work includes a detailed evaluation of the successful packet reception and packet error ratios by distinguishing between packets that are received in the absence of interference, packets that are received despite the presence of interference, as well as between packets being dropped due to channel effects, and packets being dropped due to interference. Such a differentiation is however not the objective of the work presented in this paper.

\section{IEEE 802.11P AND VEHICULAR RADIO CHANNELS}

As mentioned in the introduction, the vehicle-2-vehicle $(\mathrm{V} 2 \mathrm{~V})$ radio propagation channel is time- and frequencyselective. These characteristics stem from the pronounced multi-path propagation effects that are noticable when using a carrier frequency of $5.9 \mathrm{GHz}$ : the radiated signal is reflected and scattered by surrounding objects - e.g. other vehicles, buildings, sign posts, or trees - and arrives at a potential receiver in terms of multiple echos. Due to the different propagation distances, each path exhibits its own power attenuation, phase shift and propagation delay. The sum of all those paths then defines the (instantaneous) impulse response of the channel and is typically described by the so called power delay profile (PDP). Although it is not the objective of this paper to provide a complete tutorial on wireless propagation channels, it is important to state that a variance (or spread) of the propagation delays as small as possible is usually desired.

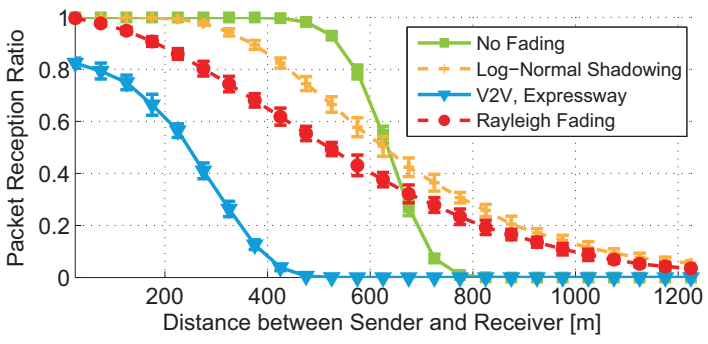

(a) Successful Packet Reception

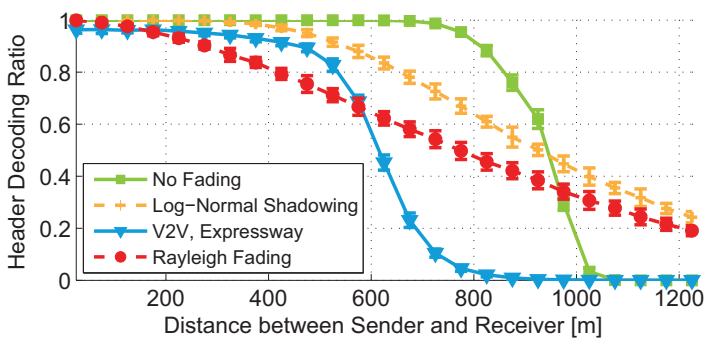

(b) Successful Header Decode

Fig. 1. Illustration of the packet reception and header decoding ratio w.r.t. the distance between a single sender and a single receiver.

If, in addition to multi-path propagation effects, transmitting and receiving vehicles are mobile, i.e. moving towards (or away from) each other, a Doppler (or frequency) shift can be observed over subsequent channel impulse responses. Similar to the PDP, a small variance (or spread) of the observed Doppler shifts is again preferred.

According to the results of recent channel measurement campaigns [1], the observed Doppler and delay spreads are, however, not sufficiently small but large, with the result that the wireless channel for safety-related inter-vehicle communications is best described through a wideband stochastic channel model, e.g. using the Tapped-Delay Line (TDL) approach. The six time- and frequency-selective vehicular channel models by Ingram et al. [11] are only one example for such channel models.

Figure 1 illustrates how the physical layer performance of IEEE $802.11 \mathrm{p}$ is influenced when such time- and frequencyselective channel conditions are exhibited. The shown curves, which plot the successful packet reception ratio (and the successful header decoding ratio) over the distance between a single sender and a single receiver, were obtained using the high fidelity network simulator proposed by Mittag et al. in [5]. The sending node transmitted 1000 packets using a data rate of $6 \mathrm{Mbps}$, a channel spacing of $10 \mathrm{MHz}$, a packet size of 400 bytes, and a transmission power of $20 \mathrm{dBm}$. The wireless channel was configured to reflect either (i) a deterministic distance decaying path loss with a reference path loss of $59.7 \mathrm{~dB}$ (at $1 \mathrm{~m}$ distance) and a path loss exponent of 1.85 , (ii) a deterministic path loss (configured as before) plus an uncorrelated shadowing using a Log-Normal shadowing with $\sigma=3.2 \mathrm{~dB}$, (iii) a deterministic path loss (configured as before) plus a fast-fading of the signal using the expressway 
oncoming characterization by Ingram et al. [11], or (iv) a deterministic path loss (configured as before) plus a Rayleigh fading using the Jakes Doppler spectrum. The considered relative speed of the vehicles was set to $200 \mathrm{~km} / \mathrm{h}$.

As can be observed in this figure, the packet reception ratio is worse than the successful header decoding ratio. The reason is quite simple: the payload is encoded using a higher order modulation scheme, namely QPSK. In contrast, the header is encoded using BPSK. As a result, the required signal-to-noise ratio (SNR) to successfully decode the header is lower than the threshold required to successfully decode the payload. Hence, an ongoing transmission can successfully be detected even if not all data symbols are successfully decodable. In case of the non-fading channel configuration, the difference between successful header decoding and successful packet reception is (more or less) linear with respect to the achieved range (approx. 200 meters gain). Similar observations can be made for the Log-Normal shadowing and the Rayleigh fading configurations. Only in the vehicle-to-vehicle expressway oncoming channel setup - which employs a TDL-based approach - the difference is non-linear: whereas a successful packet reception at very close distances is possible in only $80 \%$ of the cases, a successful header decode is possible in nearly all cases up to a distances of $500 \mathrm{~m}$. It can also be observed that the successful header decoding performance is better than in a Rayleigh fading setup up to a distance of approx. $600 \mathrm{~m}$.

The interpretation and generalization of the result shown in Figure 1 has to be carried out carefully. The TDL-based characterization of the expressway oncoming model is based on channel soundings in which sender and receiver were located more than $300 \mathrm{~m}$ away from each other. Hence, the characteristics are only valid beyond this range, and applying the same characteristics to distances below $300 \mathrm{~m}$ is very conservative and semantically inappropriate, i.e. not reflecting the performance one would observe in a real system. A similar argumentation can be applied if a Rayleigh fading at close distances is assumed: at such distances a strong line-of-sight path will most likely dominate the received signal and lead to a better performance as depicted in Figure 1. Due to this reason, a Rayleigh fading channel is used as a conservative setup in the following evaluation of CSMA.

\section{Evaluation}

In the following subsections, we briefly describe the applied evalution methodology, the considered inter-vehicle communications scenario (as well as its configuration), and finally present and discuss the obtained results.

\section{A. Methodology and Performance Metrics}

To answer whether CSMA is able to coordinate periodic broadcast transmissions in vehicular radio channels effectively, it is required to quantify the number of cases in which CSMA achieves its objective and the number of cases in which it fails to achieve its objective. That is, we need to evaluate the "lifetime" of all transmitted packets and check whether CSMA was able to suppress concurrent transmission during

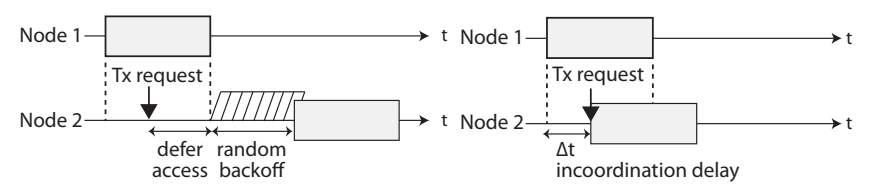

(a) Successful Coordination

(b) Failed Coordination

Fig. 2. Illustration of successful and failed coordination when using CSMA: a) if node 2 is able to sense the transmission of node 1 , it waits until node 1 has finished and then performs a random backoff. b) if node 2 is not able to sense the transmission of node 1 it, will access the channel immediately.

each transmission period successfully. We expect to observe the two cases depicted in Figure 2: (a) either all nodes (in the depicted case only node 2) are able to sense the transmission, defer a potential transmission request, and eventually backoff, or (b) one of the nodes is not able to sense the ongoing transmission and interferes with a small time delay $\Delta t$.

Since we are interested primarily in coordination failures, we quantify the probability that at least one neighboring node starts to broadcast a periodic beacon message during the transmission period of the packet under investigation, a metric termed Packet Level Incoordination in the following.

Packet Level Incoordination, PLI - The packet level incoordination, as observed from the perspective of a node $r$ and one of its generated packets $p$, describes the probability that at least one node $s, s \neq r$, transmitted a packet $q$ during the transmission period of $p$.

Compared to the original definition of PLI by TorrentMoreno et al. in [2] which is given from a receiver perspective, the above definition is not restricted to CSMA-based mechanisms and includes also the cases in which two nodes start their transmissions exactly at the same point in time. Hence, the above definition includes all possible cases of incoordination. Since it is important to differentiate between an incoordinated node located next to the reference node and an incoordinated node located further away, the PLI is evaluated with respect to the range within which incoordinated nodes are considered.

Considering the case that MAC does not perform any coordination at all, i.e. every vehicle broadcasts its periodic beacon message whenever the application generates it, the PLI can be calculated analytically as follows: the beaconing rate $r$ determines the time period $T$ during which all nodes will transmit one beacon message each, i.e.

$$
T=\frac{1}{r}
$$

With $t_{d}$ being the transmit duration of a single packet, the number of packets (or "time slots") $S$ that fit into $T$ without any overlap is then given by

$$
S=\left\lfloor\frac{T}{t_{d}}\right\rfloor
$$

and the probability that exactly $i$ out of $N$ nodes start a transmission during one of these time slots is given by

$$
P_{i}=\left(\begin{array}{c}
N \\
i
\end{array}\right)\left(\left(\frac{1}{S}\right)^{i}\left(1-\frac{1}{S}\right)^{N-i}\right)
$$


By summing up the probabilities of all $P_{i}$ with $0<i \leq N$, the overall probability $P$ that at least one out of $N$ considered nodes starts a transmission during one of the available time slots is obtained, i.e.

$$
P=\sum_{i=1}^{N} P_{i}
$$

Note that the above derivation is based on the assumption that the transmission times of the nodes are uniformly distributed. Despite the usage of the term "time slots" in the above calculation, it can also be applied to the unslotted case. In the following, it will be used to put the coordination performance of CSMA into context.

Apart from the quantification of the PLI, it is also important to resolve the type of incoordination. With respect to CSMA, the reason for an incoordination could be that either both nodes started their transmission at exactly the same point in time, e.g. due to simultaneous expiration of backoff timers, or that the incoordinated node did not sense the reference transmission, e.g. due to shadowing or fading channel characteristics.

Incoordination Delay Profile, IDP - The incoordination delay profile describes the probability distribution of the starting time differences between a set of packet transmissions $P=\left\{p_{1}, \ldots, p_{n}\right\}$ and each packet's corresponding set of incoordinated transmissions $Q_{i}=\left\{q_{i 1}, \ldots, q_{i j}\right\}, 1 \leq i \leq n$.

In case of CSMA and deterministic channel conditions, the IDP should indicate that all incoordinated nodes located within the carrier sense range - the range within which virtual or physical carrier sensing will be successful — transmit more or less simultaneously with the reference node. Only incoordinated nodes outside the carrier sense range should show significantly greater delays. Again, to determine the effectivity of CSMA with respect to this controlled spatial reuse of the channel, the IDP is evaluated with respect to the distance between sender and incoordinated node as well.

\section{B. Scenario Configuration}

The performance of CSMA is characterized using a simple highway scenario in which vehicles are placed uniformly on a $5 \mathrm{~km}$ long road with 2 lanes per direction. The highway environment is chosen since considerably high velocities, and hence pronounced fast-fading radio propagation characteristics, can be expected in this setting. A simple broadcast application that is running on each vehicle generates periodic awareness messages at an average rate $r$ (in $\mathrm{Hz}$ ). The starting time of each application is selected randomly (using a uniform distribution) from the time interval $1 / r$ seconds. Further, in order to introduce a small amount of randomness, a small jitter is applied to the interval between two subsequent awareness messages.

To evaluate different network saturation levels, application specific impact factors are varied over a reasonable parameter range, i.e. the beaconing rate is set to either $2 \mathrm{~Hz}, 5 \mathrm{~Hz}$ or $10 \mathrm{~Hz}$, the transmission power is set to either $5 \mathrm{dBm}, 10 \mathrm{dBm}$, $15 \mathrm{dBm}$ or $20 \mathrm{dBm}$, and the size of an awareness message is set to 200 bytes or 400 bytes. Furthermore, three different average vehicle densities in the range of 40 to 120 vehicles per highway kilometer are considered to vary the number of transceivers for which concurrent access has to be coordinated. Although mobility is considered in order to simulate fastfading channel conditions, vehicles are configured to keep their (initial) positions. Since CSMA does not employ any slot reservation technique, and vehicles do not alter their positions significantly during a few miliseconds (with respect to the dimension of the network in terms of communication range), the topology of the network can be considered stationary during the channel contention period. This configuration should therefore not affect the relevance of the obtained results. Nevertheless, in order to compute fast-fading propagation characteristics, a (fake) mobility of $100 \mathrm{~km} / \mathrm{h}$ is considered by radio propagation models.

With respect to IEEE 802.11p medium access control, a basic DCF with a CCA busy (i.e. physical carrier sense) threshold of $-91 \mathrm{dBm}$, a fixed contention window size of 15 slots, and a slot time of $13 \mu \mathrm{s}$ is used. Further, each vehicle is configured to use a data rate of $6 \mathrm{Mbps}$ in a $10 \mathrm{MHz}$ channel at a carrier frequency of $5.9 \mathrm{GHz}$. Receivers also make use of packet capture capabilities using a capture threshold of $8 \mathrm{~dB}$. The background noise level has been set to $-99 \mathrm{dBm}$. Note that perfect omni-directional antennas and no antenna gains are considered in this study. The introduction of a positive or negative gain would only affect the maximum distance at which a signal can be received successfully, but not the "essence" of the results.

Most importantly, the radio propagation conditions are varied as well. Initially, only a distance decaying deterministic path loss is considered to study the coordination performance in the absence of any channel fading characteristics. Such a consideration enables the identification of the fundamental CSMA weaknesses, and serves as a reference when analyzing the results of the subsequent simulations in which fading is considered. As proposed by Kunisch et al. in [12], a power law model with a reference loss of $59.7 \mathrm{~dB}$ (at $1 \mathrm{~m}$ distance) and a path loss exponent of 1.85 is used. Combined with a $20 \mathrm{dBm}$ transmit power setting, that setup yields a communication range of approx. $750 \mathrm{~m}$. Then, uncorrelated shadowing effects in terms of a Log-Normal distribution with $\sigma=3.2 \mathrm{~dB}$ are introduced. In a last step, the effect of a small-scale fading is analyzed through the simulation of a Rayleigh fading channel using the Jakes Doppler spectrum (instead of the Log-Normal shadowing).

In total, the combination of all configuration parameters yields a set of 288 different simulation experiments. To achieve statistical significance, each experiment is repeated 20 times, whereas each repetition starts with a different seed for the random number generator, and new controlled but randomly chosen positions for all vehicles. To be precise, only the applied random jitter is re-calculated. The average spacing between two vehicles remains fixed for a given vehicular density. In each experiment, twelve vehicles located in the center of the scenario are selected as reference nodes. During the simulation of an experiment, each packet transmitted by 


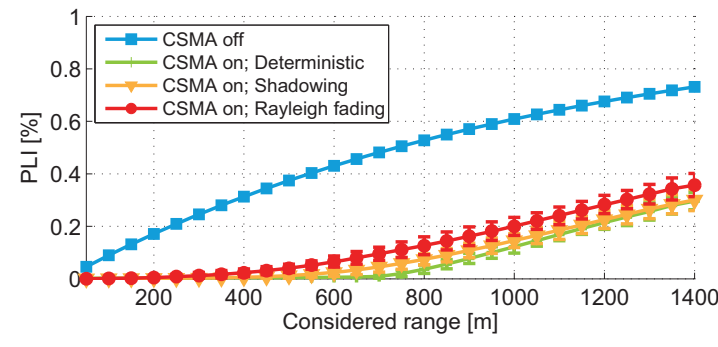

(a) 80 vehicles $/ \mathrm{km}$

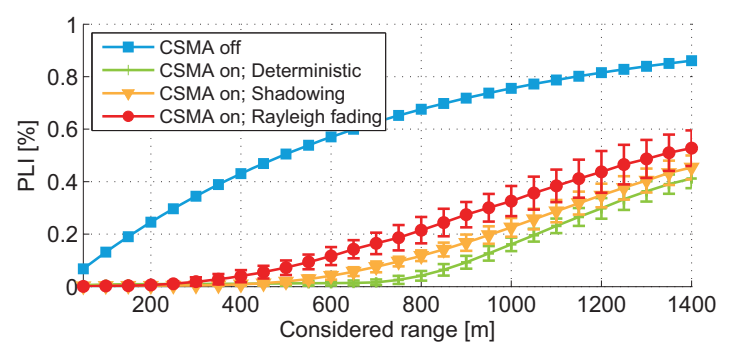

(b) 120 vehicles $/ \mathrm{km}$

Fig. 3. Probability of packet level incoordination w.r.t. considered range from transmitter: a) shows the PLI for a 80 vehicles $/ \mathrm{km}$ setup, and b) for a 120 vehicles $/ \mathrm{km}$ setup. In both setups different channel conditions have been evaluated, and compared to the case when CSMA is disabled.

any of these reference nodes is monitored and evaluated with respect to the performance metrics described in Section IV-A. Since the experiments differ with respect to the transmission rate used, e.g. some experiments use $2 \mathrm{~Hz}$ while others use $10 \mathrm{~Hz}$, the simulated time is chosen such that the same number of packets is transmitted (and evaluated) in each experiment.

Due to space restrictions, we include only the results of the 80 vehicles $/ \mathrm{km}$ and 120 vehicles/km setup in this paper, using a transmission power of $20 \mathrm{dBm}$, a packet size of 400 bytes and a beacon generation rate of $10 \mathrm{~Hz}$. These are also the most challenging scenarios with an offered load equivalent to $65 \%$ or $95 \%$ saturation of the maximum channel capacity. The results of all other scenarios (as well as the complete source code of the experiments) can be accessed online [13].

\section{Results}

Figure 3 starts with the illustration of the observable PLI probabilities in the 80 vehicles $/ \mathrm{km}$ and 120 vehicles $/ \mathrm{km}$ setups. The vehicles were configured to use a transmission power of $20 \mathrm{dBm}$, a packet size of 400 bytes, and a beaconing rate of $10 \mathrm{~Hz}$. In addition to the simulation-based results, Figure 3 shows the corresponding (analytically calculated) PLI probabilites for a CSMA-disabled setup as well (using Equation 4).

As can be seen in the 80 vehicles/km case, cf. Figure 3(a), the probability of incoordination increases rapidly with an increase of the considered range when CSMA is disabled. If CSMA is enabled and deterministic propagation conditions (i.e. no fading) are considered, the probability of incoordination remains close to zero within the communication range of approx. $750 \mathrm{~m}$. This is expected, since every node within the communication range will be blocked during a transmission,

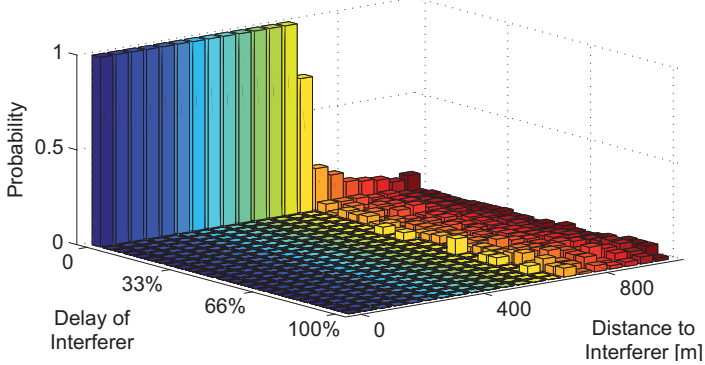

(a) No Fading

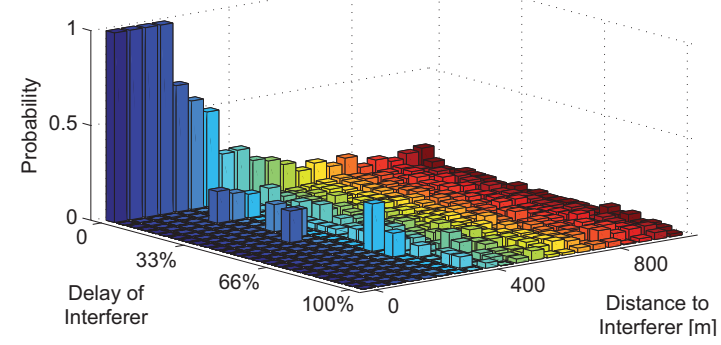

(b) Log-Normal Shadowing

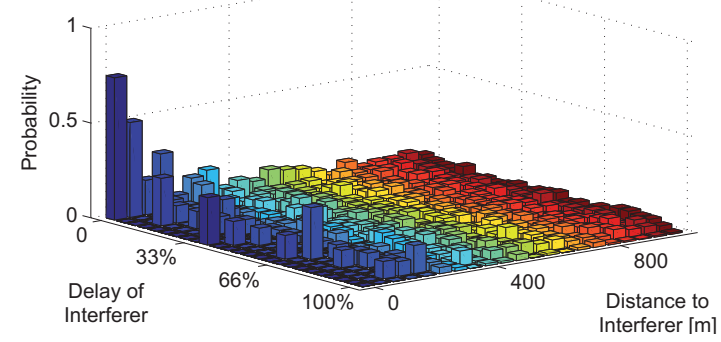

(c) Rayleigh Fading

Fig. 4. Illustration of the incoordination delay profiles of the 120 vehicles $/ \mathrm{km}$ setup, and three different channel conditions.

and the only source of incoordination can be simultaneous transmission times. The situation changes if a Log-Normal shadowing is considered in addition: the PLI increases slightly within the deterministic communication range. In case of worse fading conditions, i.e. a Rayleigh fading, incoordination probabilities increase even further. Similar observations can be made in the 120 vehicles/km scenario, cf. Figure 3(b), with the difference that the observed PLI increase due to fading is more significant. Very high channel saturation levels should therefore be avoided - which will be the case according to recent U.S. and European standardization efforts that address decentralized congestion control mechanisms.

To resolve the reasons for incoordinated transmissions, the collected IDPs with respect to the distance between the reference node and an incoordinated node have to be evaluated. Due to space restrictions, only the IDPs for the 120 vehicles $/ \mathrm{km}$ setup are shown in the following.

If a deterministic channel configuration is considered, cf. Figure 4(a), the IDPs illustrate very nicely that CSMA achieves its design objective: incoordinated transmission from within the deterministic communication range of the reference 
node happen only due to identical transmission times. Hence, the time delay $\Delta t$ between the reference transmissions and the incoordinated transmissions is always zero. As soon as the distance between the reference node and an incoordinated node is greater than the communication range, a uniform distribution of $\Delta t$ can be observed. That is also no surprise since all nodes outside of the communication range are hidden terminals.

When simulating an additional Log-Normal shadowing effect, cf. Figure 4(b), the hidden terminal problem increases, in the sense that even nodes within the deterministic communication range do not sense an ongoing transmission. As a consequence, identical transmission times are not the only reason for incoordinated transmissions from within the deterministic communication range anymore, i.e. $\Delta t$ values greater than zero are observed. A similar, but more pronounced, observation can be made when a Rayleigh fading channel is considered, cf. Figure 4(c).

To summarize the observed results: fading channel conditions reduce the coordination effectiveness of CSMA only slightly if the offered load is controlled and remains below a certain threshold, e.g. below two thirds of the maximum channel capacity. Significant differences can only be observed if the load exceeds this threshold, that is, if the maximum channel saturation level is approached.

It should be noted that the assumption of a Rayleigh fading at close distances is rather unrealistic, since a line of sight propagation path will most likely dominate the multi-path propagation in these situations. Hence, the effectiveness of CSMA should be better in reality, i.e. the PLI curve is expected to be somewhere between the Log-Normal shadowing and the Rayleigh fading setup. Hence, we feel confident to conclude that the initially raised question, i.e. will CSMA be able to effectively suppress concurrent transmissions by neighboring nodes, can be answered with a "yes".

\section{Conclusions}

In this paper, we evaluated the ability of CSMA to effectively coordinate multiple access in vehicular radio channels. In particular, we evaluated whether CSMA is able to effectively suppress overlapping packet transmissions by neighboring vehicles in a highway scenario. To incoorporate the effects that can be observed at the wireless channel and the physical layer appropriately, a high fidelity network simulator has been used. The deployed simulator emulates the signal processing steps of a real transceiver and models the multipath propagation effect of the wireless channel in detail.

The obtained results show that CSMA is able to coordinate multiple access effectively (and as desired) if no fading conditions are exhibited: simultaneous transmissions (due to hidden terminal situations) are carried out primarily by vehicles outside of the (deterministic) communication range. If fading conditions are introduced, the effectiveness of CSMA is slightly reduced, in the sense that overlapping transmissions (due to hidden terminal situations) are carried out also by vehicles located within the (deterministic) communication range. Nevertheless, it can be stated that the reduction remains small as long as the communication system is not operated at the limit of the available channel capacity $(6 \mathrm{Mbps}$ in this case). Whether one is willing to tolerate this reduction is of course subject to discussions.

It should be noted, that we did not study the case in which a correlated shadowing is exhibited. Such a shadowing is observed whenever buildings or other vehicles obstruct the propagation of a transmitted signal and prohibit the successful detection (and reception) of an ongoing transmission. Although such conditions will only apply to a subset of the communication "links" (and not to all "links" between a single sender and all of its neighbors), it might be possible that such conditions will affect the coordination performance as well. Whether this is the case is subject to future work.

\section{REFERENCES}

[1] C. Mecklenbräuker, A. Molisch, J. Karedal, F. Tufvesson, A. Paier, L. Bernado, T. Zemen, O. Klemp, and N. Czink, "Vehicular Channel Characterization and Its Implications for Wireless System Design and Performance," Proceedings of the IEEE, vol. 99, no. 7, pp. 1189-1212, July 2011.

[2] M. Torrent-Moreno, S. Corroy, F. Schmidt-Eisenlohr, and H. Hartenstein, "IEEE 802.11-based One-hop Broadcast Communications: Understanding Transmission Success And Failure Under Different Radio Propagation Environments," in Proceedings of the 9th ACM international symposium on Modeling analysis and simulation of wireless and mobile systems, ser. MSWiM '06. New York, NY, USA: ACM, 2006, pp. 68-77.

[3] K. Bilstrup, E. Uhlemann, E. G. Ström, and U. Bilstrup, "On the Ability of the 802.11p MAC Method and STDMA to Support RealTime Vehicle-to-Vehicle Communication," EURASIP Journal on Wireless Communication Networks, vol. 2009, January 2009.

[4] K. Bilstrup, E. Uhlemann, and E. G. Ström, "Scalability Issues of the MAC Methods STDMA and CSMA of IEEE 802.11p When Used in VANETs," in 2010 IEEE International Conference on Communications Workshops (ICC). IEEE, May 2010, pp. 1-5.

[5] J. Mittag, S. Papanastasiou, H. Hartenstein, and E. Ström, "Enabling Accurate Cross-Layer PHY/MAC/NET Simulation Studies of Vehicular Communication Networks," Proceedings of the IEEE, vol. 99, no. 7, pp. $1311-1326$, july 2011.

[6] F. Tobagi and L. Kleinrock, "Packet Switching in Radio Channels: Part II-The Hidden Terminal Problem in Carrier Sense Multiple-Access and the Busy-Tone Solution," IEEE Transactions on Communications, vol. 23, no. 12 , pp. 1417- 1433, Dec. 1975.

[7] G. Bianchi, "Performance Analysis of the IEEE 802.11 Distributed Coordination Function," IEEE Journal on Selected Areas in Communications, vol. 18, no. 3, pp. 535-547, Mar. 2000.

[8] N. Gupta, "A Performance Analysis of the 802.11 Wireless Lan Medium Access Control," Communications in Information \& Systems, vol. 3, no. 4, pp. 479-304, 2003.

[9] M. Torrent-Moreno, D. Jiang, and H. Hartenstein, "Broadcast Reception Rates and Effects of Priority Access in 802.11-based Vehicular Ad-hoc Networks," in Proceedings of the 1st ACM international workshop on Vehicular ad hoc networks, ser. VANET '04. New York, NY, USA: ACM, 2004, pp. 10-18.

[10] F. Schmidt-Eisenlohr, "Interference in Vehicle-to-Vehicle Communication Networks: Analysis, Modeling, Simulation and Assessment," Ph.D. dissertation, Karlsruhe Institute of Technology (KIT), Karlsruhe, 2010.

[11] G. Acosta-Marum and M. Ingram, "Six Time- and Frequency-Selective Empirical Channel Models for Vehicular Wireless LANs," Vehicular Technology Magazine, IEEE, vol. 2, no. 4, pp. 4-11, 2007.

[12] J. Kunisch and J. Pamp, "Wideband Car-to-Car Radio Channel Measurements and Model at $5.9 \mathrm{GHz}$," in Vehicular Technology Conference, 2008. VTC 2008-Fall. IEEE 68th. IEEE, Sep. 2008, pp. 1-5.

[13] J. Mittag, "Characterization, Avoidance and Repair of Packet Collisions in Inter-Vehicle Communication Networks: The Complete Set of Results," February 2012, http://dsn.tm.kit.edu/download/ns3physim/results.html. 